\title{
Shaping lattice through irrelevant perturbation: Ising model
}

\section{Armen Poghosyan}

Yerevan Physics Institute,

Alikhanian Br. 2, AM-0036 Yerevan, Armenia

E-mail: armenpoghos@yerphi.am

ABSTRACT: The leading irrelevant perturbation, which controls the deviation of critical square lattice Ising model with periodic boundary conditions from its continuous CFT ana$\log$ is identified. An explicit expression for the coupling constant in terms of the anisotropy parameter is found. We calculate the next to leading $\sim 1 / N^{2}$ corrections to the spectrum on both lattice theory and the perturbed CFT sides for several classes of states, always getting exact agreement. We discuss also how the perturbing operators and the higher integrals of motion are related.

KEywords: Conformal Field Theory, Lattice Integrable Models, Field Theories in Lower Dimensions, Conformal and W Symmetry

ARXiv EPrint: 1908.06291 


\section{Contents}

1 Introduction 1

2 Eigenvalues of the transfer matrix $\quad 2$

2.1 Leading Eigenvalues 5

2.2 CFT prediction 5

2.3 Large $N$ expansion of leading eigenvalues 5

$\begin{array}{ll}2.4 & \text { Partition function on a large torus and Ising fermions }\end{array}$

3 Irrelevant perturbation of Ising CFT $\quad 8$

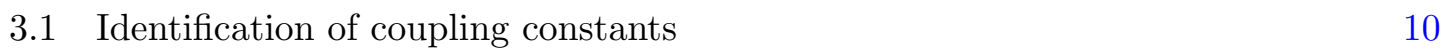

3.2 Energy corrections for the states $L_{-1}^{p-1} \bar{L}_{-1}^{\tilde{p}-1}|1 / 2 ; 1 / 2\rangle \quad 11$

3.3 Energy corrections for the states $\psi_{-p} \bar{\psi}_{-\tilde{p}}|1 / 16 ; 1 / 16\rangle$

$\begin{array}{ll}3.4 & \text { An example of doubly degenerate states } \\ \end{array}$

4 Summary and discussion $\quad 14$

\section{Introduction}

It is well known that large scale behavior of many statistical systems near their critical points can be described by (Euclidean) Quantum Field Theories (QFT) (see e.g. [1]). In such description many microscopic details of initial theory are washed out, so that various statistical systems may lead to the same continuous theory. In this respect it is interesting to investigate intermediate scales, where some subleading corrections (besides leading finite size effects) to the QFT description still are noticeable. Then it would be possible to clarify how specific microscopic structure of the system is reflected in this corrections. From QFT point of view such corrections can be described as perturbations by irrelevant operators which are allowed to carry nonzero spins, since the rotational invariance at small distances is violated $[2,3]$. In this respect the two dimensional Ising model [4] is an ideal object to investigate, since it is exactly integrable [5]. Moreover, for some boundary conditions all the eigenvalues of its transfer matrix are known exactly even for finite lattices (see e.g. [6] for toroidal boundary conditions, [7] for several other cases).

The paper is organized as follows.

In section 2 we systematically investigate the eigenvalues of periodic critical Ising transfer-matrix in large $L$ limit ( $L$ is the number of spins in a horizontal row). We show how $1 / L$ terms exactly match with CFT prediction and compute the next $1 / L^{3}$ corrections for certain families of eigenvalues. The $1 / L^{3}$ corrections under discussion describe breakdown of rotational invariance due to lattice artifacts. 


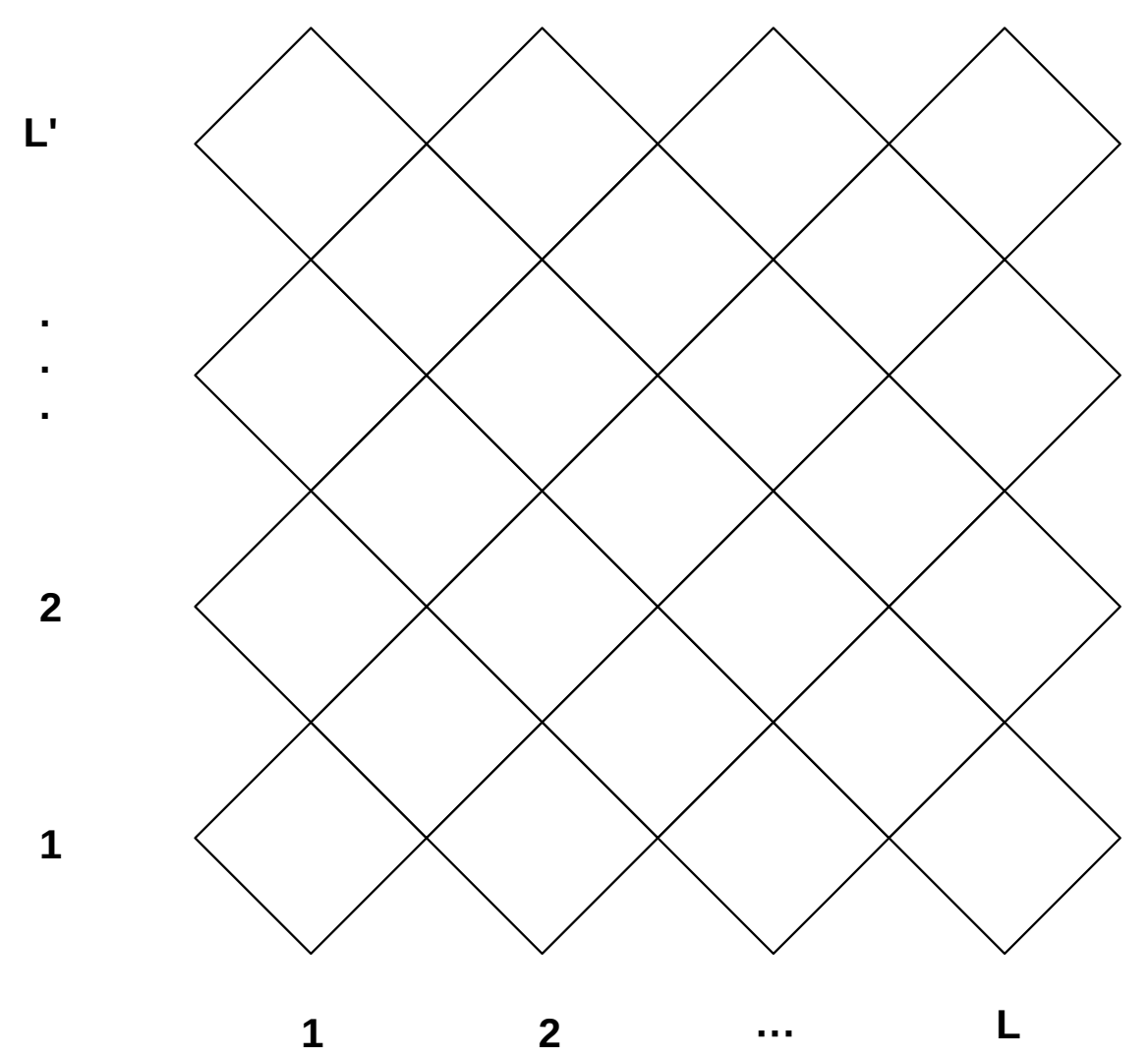

Figure 1. Periodic Ising Lattice. The leftmost (lowermost) spins are identified with corresponding rightmost (uppermost) spins.

In section 3 we address the question, how to perturb Ising CFT in order to get precisely those corrections which we obtained investigating lattice model. We were able to identify the perturbing fields and the respective coupling constants. Namely we show that the perturbing fields are the spin 4 current and its antiholomorphic counterpart, which were introduced in the context of integrable structure of CFT long ago. Having non-zero spin, these fields break the rotational invariance, while being irrelevant, they slightly correct the large distance behavior in a way, to mimic the lattice result.

Finally we end up with a summary of our results and discuss the possibility of generalization for full spectrum and for higher order corrections.

\section{Eigenvalues of the transfer matrix}

Consider square lattice Ising model. We'll adopt the " 45 degree rotated" version presented in great details e.g. in Baxter's seminal book [6]. The lattice consists of $L$ vertical columns of $L^{\prime}$ faces, or equivalently, of $L^{\prime}$ horizontal rows of $L$ faces (see figure 1 ). We will consider periodic boundary condition in both, horizontal and vertical directions, so that the $L+1$-th column is identified with the first column and the $L^{\prime}+1$-th row with the first one. The 

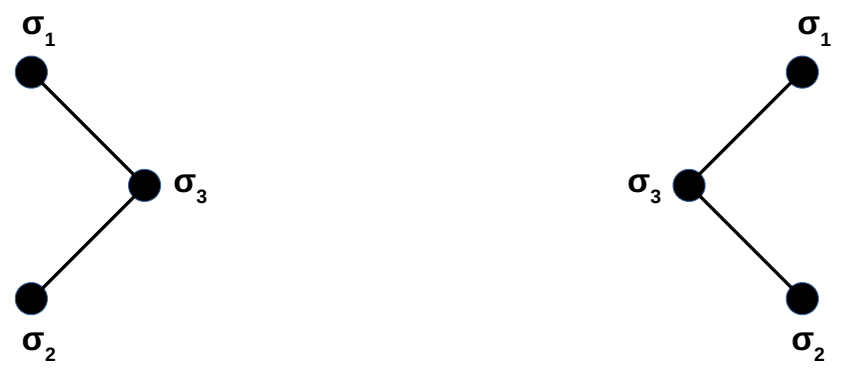

Figure 2. Two basic configurations of three Ising spins.

partition function of the theory is given by

$$
Z=\sum_{\{\sigma\}} \exp \left(J \sum_{\langle i, j\rangle} \sigma_{i} \sigma_{j}+K \sum_{\langle k, l\rangle} \sigma_{i} \sigma_{j}\right),
$$

where $\langle i, j\rangle$ belong to the set of SW-NE, and $\langle k, l\rangle$ to the NW-SE edges. In what follows, we will restrict ourselves to the case of critical Ising model. The well known criticality condition $\sinh 2 J \sinh 2 K=1$ can be conveniently parameterized by a single parameter $u$ by setting

$$
\sinh 2 J=\cot 2 u ; \quad \sinh 2 K=\tan 2 u,
$$

with $0<u<\pi / 4$. Sometimes the parameter $u$ is referred as the anisotropy. The value $u=\pi / 8$ corresponds to the isotropic case.

To define the transfer matrix of the model, let us denote the Boltzmann weights of two basic types of three spin configurations shown in figure 2 as

$$
W\left(\begin{array}{ll}
\sigma_{1} & \sigma_{3} \\
\sigma_{2} &
\end{array}\right) \quad \text { and } \quad W\left(\begin{array}{c}
\sigma_{3} \\
\sigma_{1} \\
\sigma_{2}
\end{array}\right)
$$

where $\sigma_{i}= \pm 1$ are the Ising spins. Thus, the arrangement of spins in the argument of $W$ follows to the geometric pattern of their locations on the lattice. These Boltzmann weights are explicitly given by

$$
\begin{gathered}
W\left(\begin{array}{c}
\sigma \\
\sigma
\end{array}\right)=W\left(\begin{array}{c}
\sigma \\
\sigma
\end{array}\right)=\sqrt{2} \cos (u) \cos \left(\frac{\pi}{4}-u\right), \\
W\left(\begin{array}{c}
\sigma \\
\sigma
\end{array}-\sigma\right)=W\left(\begin{array}{r}
\sigma \\
-\sigma
\end{array}\right)=\sqrt{2} \sin (u) \sin \left(\frac{\pi}{4}-u\right), \\
W\left(\begin{array}{r}
\sigma \\
-\sigma
\end{array}\right)=W\left(\begin{array}{r}
\sigma \\
\sigma
\end{array}\right)=\sqrt{2} \sin (u) \cos \left(\frac{\pi}{4}-u\right) \\
W\left(\begin{array}{r}
-\sigma \\
\sigma
\end{array}\right)=W\left(\begin{array}{r}
\sigma \\
\sigma
\end{array}\right)=\sqrt{2} \sin \left(\frac{\pi}{4}-u\right) \cos (u),
\end{gathered}
$$

where $\sigma= \pm 1$ and $u$ is the anisotropy parameter. Note that to get (2.3) from (2.2), one should include an overall extra factor $\frac{1}{2} \sqrt{\sin 4 u}$. Such shift in vacuum energy is convenient particularly because the transfer matrix, defined below, becomes the one-step shift operator at the values $u=0$ and $u=\frac{\pi}{4}$. 


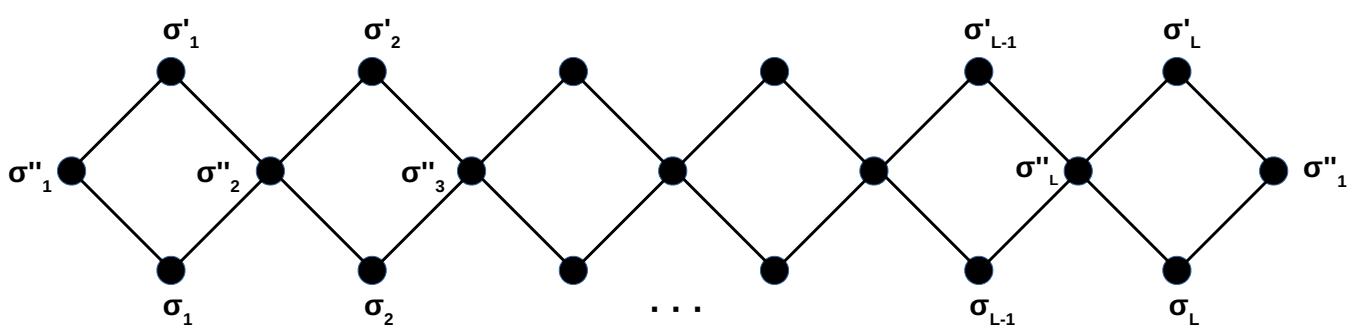

Figure 3. The transfer matrix. Sum over all middle spins $\sigma^{\prime \prime}$ is assumed.

After imposing periodic boundary condition in horizontal direction for the transfermatrix we get (See figure 3)

$$
T_{\sigma, \sigma^{\prime}}=\sum_{\sigma^{\prime \prime}} W\left(\begin{array}{cc}
\sigma_{1}^{\prime \prime} & \sigma_{1}^{\prime} \\
& \sigma_{1}
\end{array}\right) W\left(\begin{array}{cc}
\sigma_{1}^{\prime} & \sigma_{2}^{\prime \prime} \\
\sigma_{1} &
\end{array}\right) W\left(\begin{array}{cc}
\sigma_{2}^{\prime \prime} & \sigma_{2}^{\prime} \\
& \sigma_{2}
\end{array}\right) \cdots W\left(\begin{array}{ll}
\sigma_{L}^{\prime} & \sigma_{1}^{\prime \prime} \\
\sigma_{L} &
\end{array}\right)
$$

The exact eigenvalues of this transfer-matrix can be found in [6]. We will use a slightly modified version of the explicit expressions, presented in [7] based on more general treatment of [8] (see also later works [9-11]). There are two sets of eigenvalues, distinguished by the parameter $r= \pm 1$ and denoted by $\Lambda^{( \pm)}$respectively. To be precise, let me warn the reader, that the quantities $\Lambda^{( \pm)}$introduced below, actually are the square roots of transfer matrix eigenvalues. Nevertheless with a slight abuse of terminology, they will be referred simply as eigenvalues.

For $r=1$ the set of eigenvalues are given explicitly as [7] $(\lfloor x\rfloor$ stands for the largest integer not exceeding $x$ ):

$$
\begin{aligned}
\Lambda^{(+)}\left(\mu_{k}, \bar{\mu}_{k}\right)= & \sqrt{2}\left(2 e^{2 i u+\frac{i \pi}{4}}\right)^{-L} \prod_{k=1}^{\left\lfloor\frac{L}{2}\right\rfloor}\left(i \mu_{k} \tan \left(\frac{\pi(2 k-1)}{4 L}\right)+e^{4 i u}\right) \\
& \times \prod_{k=1}^{\left\lfloor\frac{L+1}{2}\right\rfloor}\left(i \cot \left(\frac{\pi(2 k-1)}{4 L}\right)+e^{4 i u} \bar{\mu}_{k}\right),
\end{aligned}
$$

where $\mu_{k}, \bar{\mu}_{k} \in\{+1,-1\}$ are subject to the constraint

$$
\prod_{k=1}^{\left\lfloor\frac{L}{2}\right\rfloor} \mu_{k} \prod_{k=1}^{\left\lfloor\frac{L+1}{2}\right\rfloor} \bar{\mu}_{k}=1
$$

For $r=-1$ we have another set of eigenvalues [7]:

$$
\begin{aligned}
\Lambda^{(-)}\left(\mu_{k}, \bar{\mu}_{k}\right)= & \sqrt{1+L}\left(2 e^{2 i u+\frac{i \pi}{4}}\right)^{-L} \prod_{k=1}^{\left\lfloor\frac{L}{2}\right\rfloor}\left(i \mu_{k} \tan \left(\frac{\pi k}{2 L}\right)+e^{4 i u}\right) \\
& \times \prod_{k=1}^{\left\lfloor\frac{L-1}{2}\right\rfloor}\left(i \cot \left(\frac{\pi k}{2 L}\right)+e^{4 i u} \bar{\mu}_{k}\right) ;
\end{aligned}
$$

where again $\mu_{k}, \bar{\mu}_{k} \in\{+1,-1\}$, but there is no constraint anymore.

Notice that altogether we get $2^{L-1}+2^{L-1}=2^{L}$ eigenvalues, which exactly matches the size of transfermatrix (2.4). 


\section{$2.1 \quad$ Leading Eigenvalues}

The leading eigenvalues in both sectors correspond to the case when all $\mu_{k}=+1, \bar{\mu}_{k}=+1$. The expressions for these eigenvalues can be simplified and represented as

$$
\left(\Lambda_{\max }^{(+)}\right)^{2}=\prod_{k=0}^{L-1}\left(\sin (4 u) \sin \left(\frac{\pi k}{L}+\frac{\pi}{2 L}\right)+1\right)
$$

and

$$
\left(\Lambda_{\max }^{(-)}\right)^{2}=\prod_{k=0}^{L-1}\left(\sin (4 u) \sin \left(\frac{\pi k}{L}\right)+1\right)
$$

\subsection{CFT prediction}

From now on instead of parameters $L$ and $L^{\prime}$ we'll use the even integers

$$
N=2 L \quad \text { and } \quad M=2 L^{\prime},
$$

which are the numbers of vertical and horizontal "zigzagging" columns and rows of the lattice respectively (see figure 1). Conformal field theory predicts that the logarithm of transfer matrix eigenvalues in large $N, M$ limit behave as $[12,13]$

$$
M \log \Lambda \sim-\frac{N M}{2} f_{\text {bulk }}+\left(\Delta-\frac{c}{24}\right) \log q+\left(\bar{\Delta}-\frac{c}{24}\right) \log \bar{q}
$$

where $f_{\text {bulk }}$ is the free energy per site (the number of sights in our lattice is $\frac{N M}{2}$ ), $\Delta, \bar{\Delta}$ are the left and right dimensions of the conformal field which creates the corresponding eigenstate from the vacuum. The parameter $q=\exp 2 \pi i \tau$, where $\tau$ is modulus of the torus on which our 2d CFT lives, should be identified as

$$
2 \pi i \tau=\log q=-\frac{2 \pi i M e^{-4 i u}}{N}
$$

Finally, $c$ is the Virasoro central charge, which for our case of Ising model, assumes the value $c=1 / 2$.

Notice also that besides finite size scaling, CFT predicts also the universal amplitude ratios (see e.g. [14] and references therein).

\subsection{Large $N$ expansion of leading eigenvalues}

Denote

$$
f(x)=\log (1+\sin (4 u) \sin (x))
$$

and $h=\frac{2 \pi}{N}$. Due to Euler-Maclaurin summation formula $\left(B_{n}(\alpha)\right.$ are the Bernoulli polynomials) from eq. (2.8) we get

$$
\begin{aligned}
\log \Lambda_{\max }^{(+)}+\frac{N}{2} f_{\mathrm{bulk}} & =\sum_{n=1}^{\infty} \frac{B_{n}\left(\frac{1}{2}\right)\left(f^{(n-1)}(\pi)-f^{(n-1)}(0)\right) h^{n-1}}{n !} \\
& =\frac{\pi \sin (4 u)}{12 N}+\frac{7 \pi^{3}\left(2 \sin (4 u)-4 \sin ^{3}(4 u)\right)}{1440 N^{3}}+O\left(1 / N^{5}\right)
\end{aligned}
$$


and from $(2.9)$

$$
\begin{aligned}
\log \Lambda_{\text {max }}^{(-)}+\frac{N}{2} f_{\text {bulk }} & =\sum_{n=1}^{\infty} \frac{B_{n}(0)\left(f^{(n-1)}(\pi)-f^{(n-1)}(0)\right) h^{n-1}}{n !} \\
& =-\frac{\pi \sin (4 u)}{6 N}-\frac{\pi^{3}\left(2 \sin (4 u)-4 \sin ^{3}(4 u)\right)}{180 N^{3}}+O\left(1 / N^{5}\right)
\end{aligned}
$$

The bulk free energy is given by

$$
f_{\mathrm{bulk}}=-\int_{0}^{\pi} f(x) \frac{d x}{2 \pi} .
$$

Taking into account that the leading eigenvalue should correspond to the identity operator with conformal dimensions $\Delta=\bar{\Delta}=0$, consistency of the leading terms in (2.13), (2.14) with CFT prediction (2.10) immediately fixes the value of central charge $c=1 / 2$ and identifies the torus parameter $q$ with the expression (2.11). Besides for the dimensions of the conformal field corresponding to the eigenvalue $\Lambda_{\max }^{(-)}$we get $\Delta=\bar{\Delta}=1 / 16$, which are the correct dimensions of Ising spin field.

\subsection{Partition function on a large torus and Ising fermions}

For given $p$, denote $\Lambda_{p}^{(+)}\left(\bar{\Lambda}_{p}^{(+)}\right)$the r.h.s. of $(2.5)$ for the case when $r=1$ with all $\mu$ 's and $\bar{\mu}$ 's set to 1 with a single exception, namely $\mu_{p}=-1\left(\bar{\mu}_{p}=-1\right)$. Analogously, using (2.7), we define $\Lambda_{p}^{(-)}, \bar{\Lambda}_{p}^{(-)}$for the case when $r=-1$. In large $N$ limit the impact of flipping the sign at position $p$ are given by ratios

$$
\begin{aligned}
& \log \frac{\Lambda_{p}^{(+)}}{\Lambda_{\max }^{(+)}}=-\frac{i \pi(2 p-1) e^{-4 i u}}{N}-\frac{i \pi^{3}(2 p-1)^{3} e^{-4 i u}\left(1-e^{-8 i u}\right)}{12 N^{3}}+O\left(\frac{1}{N^{5}}\right) \\
& \log \frac{\bar{\Lambda}_{p}^{(+)}}{\Lambda_{\max }^{(+)}}=\frac{i \pi(2 p-1) e^{4 i u}}{N}+\frac{i \pi^{3}(2 p-1)^{3} e^{4 i u}\left(1-e^{8 i u}\right)}{12 N^{3}}+O\left(\frac{1}{N^{5}}\right)
\end{aligned}
$$

and

$$
\begin{aligned}
\log \frac{\Lambda_{p}^{(-)}}{\Lambda_{\max }^{(-)}} & =-\frac{2 i \pi p e^{-4 i u}}{N}-\frac{2 i \pi^{3} p^{3} e^{-4 i u}\left(1-e^{-8 i u}\right)}{3 N^{3}}+O\left(\frac{1}{N^{5}}\right) \\
\log \frac{\bar{\Lambda}_{p}^{(-)}}{\Lambda_{\max }^{(-)}} & =\frac{2 i \pi p e^{4 i u}}{N}+\frac{2 i \pi^{3} p^{3} e^{4 i u}\left(1-e^{8 i u}\right)}{3 N^{3}}+O\left(\frac{1}{N^{5}}\right)
\end{aligned}
$$

It is important to notice that due to factorized structure of eigenvalues (2.5) and (2.7), if there are several indices $p_{1}, p_{2}, \cdots$, such that the respective $\mu$ 's or $\bar{\mu}$ 's assume the value -1 , the logarithm of ratios of eigenvalues will be given by the same expressions (2.16) and (2.17) summed over above specified indices p.

Consider thermodynamic limit when $M, N$ are sent to infinity while keeping their ratio fixed. Notice that $q, \bar{q}$ remain finite as seen from (2.11). Keeping the leading terms only, we can conveniently rewrite expressions (2.16), (2.17) in view of eq. (2.11) as

$$
\left(\frac{\Lambda_{p}^{(+)}}{\Lambda_{\max }^{(+)}}\right)^{M} \sim q^{p-\frac{1}{2}} ;\left(\frac{\Lambda_{p}^{(-)}}{\Lambda_{\max }^{(+)}}\right)^{M} \sim q^{\bar{p}-\frac{1}{2}} ;\left(\frac{\bar{\Lambda}_{p}^{(+)}}{\Lambda_{\max }^{(-)}}\right)^{M} \sim q^{p} ;\left(\frac{\Lambda_{p}^{(-)}}{\Lambda_{\max }^{(-)}}\right)^{M} \sim \bar{q}^{p}
$$


In other words, the sign flip of $\mu_{p}\left(\bar{\mu}_{p}\right)$ in partition sum costs a multiplier $q^{p-\frac{1}{2}}\left(\bar{q}^{p-\frac{1}{2}}\right)$ in the $r=1$ sector and a multiplier $q^{p}\left(\bar{q}^{p}\right)$ in the sector $r=-1$. We'll need also the contributions of the eigenvalues $\Lambda_{\max }^{( \pm)}$in partition function. Again, dropping $O\left(1 / N^{3}\right)$ terms, from eqs. (2.13), (2.14) we easily get

$$
\left(\Lambda_{\max }^{(+)}\right)^{M} \sim e^{-M N f_{\text {bulk }} / 2}(q \bar{q})^{-\frac{1}{48}} ; \quad\left(\Lambda_{\max }^{(-)}\right)^{M} \sim e^{-M N f_{\text {bulk }} / 2}(q \bar{q})^{-\frac{1}{48}+\frac{1}{16}} .
$$

As a result, a generic eigenvalue, say in sector $r=-1$, specified by conditions $\mu_{p_{1}}=\mu_{p_{2}}=$ $\cdots=\mu_{p_{R}}=-1, \bar{\mu}_{\tilde{p}_{1}}=\bar{\mu}_{\tilde{p}_{2}}=\cdots=\bar{\mu}_{\tilde{p}_{L}}=-1$ with all other $\mu$ 's and $\bar{\mu}$ 's taking the value $1,{ }^{1}$ will contribute a term

$$
e^{-M N f_{\text {bulk }} / 2}(q \bar{q})^{-\frac{1}{48}+\frac{1}{16}} q^{p_{1}} q^{p_{2}} \cdots q^{p_{R}} \bar{q}^{\tilde{p}_{1}} \bar{q}^{\tilde{p}_{2}} \cdots \bar{q}^{\tilde{p}_{L}}
$$

Similarly, the contribution of a generic eigenvalue from the sector $r=-1$ in partition function reads

$$
e^{-M N f_{\text {bulk }} / 2}(q \bar{q})^{-\frac{1}{48}+\frac{1}{16}} q^{p_{1}-1 / 2} q^{p_{2}-1 / 2} \cdots q^{p_{R}-1 / 2} \bar{q}^{\tilde{p}_{1}-1 / 2} \bar{q}^{\tilde{p}_{2}-1 / 2} \cdots \bar{q}^{\tilde{p}_{L}-1 / 2} .
$$

Here we should keep in mind that due to the constraint (2.6), the total number of minus signs, i.e. $R+L$ must be even. Now, by elementary considerations one can get convinced that the partition function on the torus in continuum limit can be represented as

$$
\begin{array}{r}
Z \sim e^{-M N f_{\text {bulk }} / 2}(q \bar{q})^{-\frac{1}{48}} \\
\times\left(\frac{1}{2} \prod_{k=1}^{\infty}\left(1+q^{k-\frac{1}{2}}\right)\left(1+\bar{q}^{k-\frac{1}{2}}\right)\right. \\
+\frac{1}{2} \prod_{k=1}^{\infty}\left(1-q^{k-\frac{1}{2}}\right)\left(1-\bar{q}^{k-\frac{1}{2}}\right) \\
\left.+(q \bar{q})^{\frac{1}{16}} \prod_{k=1}^{\infty}\left(1+q^{k}\right)\left(1+\bar{q}^{k}\right)\right) .
\end{array}
$$

Note that the sum with factors $1 / 2$ on second line implements the constraint (2.6), since the terms with a product of odd number of minus signs get canceled.

Obviously, the form of (2.19) reflects the well known fact that the Ising model in continuous limit is the theory of free fermions. The fermionic fields are single valued in vacuum sector (manifested by the half integer modes on second line of (2.19)), while they are non-local with respect to the spin field and have integer modes as seen on third line of (2.19). Let us briefly recall few relevant facts about free fermion theory. We will simply state the results omitting details and proofs. The aim is to specify notations which will be used later on. A comprehensive review of $2 \mathrm{~d}$ fermion CFT can be found e.g. in book [15]. The left and right moving fermion fields can be defined through mode expansions

$$
\begin{aligned}
& \psi(z)=\sum_{\nu} \frac{\psi_{\nu}}{z^{\nu+\frac{1}{2}}} \\
& \bar{\psi}(\bar{z})=\sum_{\nu} \frac{\bar{\psi}_{\nu}}{\bar{z}^{\nu+\frac{1}{2}}}
\end{aligned}
$$

\footnotetext{
${ }^{1} L$, the number of parameters $\bar{\mu}$ taking the value -1 should not be confused with the number of rows of faces introduced earlier. In fact the latter does not appear in the paper any more.
} 
where sum is over all half integers or over all integers, depending whether it acts in vacuum sector (usually referred as Neveu-Schwartz sector) or on spin (Ramond) sector. The expansion modes satisfy the anticommutation relations

$$
\left\{\psi_{\nu}, \psi_{\mu}\right\}=\delta_{\nu+\mu, 0} ; \quad\left\{\bar{\psi}_{\nu}, \bar{\psi}_{\mu}\right\}=\delta_{\nu+\mu, 0}
$$

In terms of fermion modes the sector $r=1$ is spanned on states

$$
\prod_{k=1}^{\infty}\left(\psi_{-k+\frac{1}{2}}\right)^{\varepsilon_{k-\frac{1}{2}}} \prod_{k=1}^{\infty}\left(\bar{\psi}_{-k+\frac{1}{2}}\right)^{\bar{\varepsilon}_{k-\frac{1}{2}}}|0 ; 0\rangle
$$

where $|0 ; 0\rangle$ is the vacuum state with dimensions $\Delta=\bar{\Delta}=0$ and the occupation numbers $\varepsilon_{\nu}, \bar{\varepsilon}_{\nu}$ assume values 0 or 1 . The relation of (2.23) to the eigenstates of Ising transfer matrix is very simple: if an occupation number $\varepsilon_{k-1 / 2}=1\left(\varepsilon_{k-1 / 2}=0\right)$ then $\mu_{k}=-1$ $\left(\mu_{k}=1\right)$. The analogous relation holds also for quantities $\bar{\varepsilon}$ and $\bar{\mu}$. As a consequence, the constraint (2.6) requires, that the total fermion number

$$
N_{f}=\sum_{\nu}\left(\varepsilon_{\nu}+\bar{\varepsilon}_{\nu}\right) \equiv N_{L}+N_{R}
$$

must be even. Evidently there are two alternatives here. Either both $N_{L}$ and $N_{R}$ are even or both are odd. It is easy to see that the former case corresponds to the Virasoro module created from the vacuum $|0 ; 0\rangle$ while the latter one to the module created from primary state (related to the energy density) $\left|\frac{1}{2} ; \frac{1}{2}\right\rangle$.

Similarly the Ramond subspace is spanned over the states

$$
\prod_{k=1}^{\infty}\left(\psi_{-k}\right)^{\varepsilon_{k}} \prod_{k=1}^{\infty}\left(\bar{\psi}_{-k}\right)^{\bar{\varepsilon}_{k}}\left|\frac{1}{16} ; \frac{1}{16}\right\rangle
$$

which exactly match the states of the sector $r=-1$ in lattice side. It is not difficult to calculate the torus partition function of this free fermion theory

$$
Z_{\text {ferm }}=\operatorname{Tr} q^{L_{0}-\frac{c}{24}} \bar{q}^{\bar{L}_{0}-\frac{c}{24}},
$$

where $L_{0}, \bar{L}_{0}$ are left and right Virasoro 0-modes, $q$ and $\bar{q}$, as earlier, are the torus parameter and its conjugate and $c=1 / 2$ is the Virasoro central charge. The trace is over the states (2.25) and (2.23) subject to the constraint $N_{f}=0 \bmod 2$ (see eq. (2.24)). The result besides the non universal factor $\exp \left(-N M f_{\text {bulk }}\right)$ exactly matches the the partition sum (2.19).

\section{Irrelevant perturbation of Ising CFT}

Let us introduce coordinate $\zeta=x+i y$ on cylinder $(\zeta \sim \zeta+2 \pi)$, which is related to the coordinate $z$ on plane through exponential map $z=\exp \zeta$. The least irrelevant perturbation of Ising CFT from the conformal family of identity operator, responsible for deviation from conformal theory, is (see $[2,3,16,17]$ )

$$
H_{\mathrm{int}}=\int\left(g T_{c y l}^{2}(\zeta)+\bar{g} \bar{T}_{c y l}^{2}(\bar{\zeta})\right) \frac{d y}{2 \pi}
$$


where $T_{c y l}^{2}$ and its antiholomorphic counter part are regularized squares of the energy momentum tensor. More precisely

$$
T_{c y l}^{2}(\zeta) \equiv \oint \frac{T_{c y l}\left(\zeta^{\prime}\right) T_{c y l}(\zeta) d \zeta^{\prime}}{2 \pi i\left(\zeta^{\prime}-\zeta\right)}
$$

where integration over $\zeta^{\prime}$ is along a small contour surrounding $\zeta$ anticlockwise. Using transformation rule of stress-energy tensor from plain to cylinder

$$
T_{c y l}(\zeta)=z^{2} T(z)-\frac{c}{24},
$$

where the second term comes from Schwarzian derivative, one can see that in terms of conventional Virasoro modes the interaction Hamiltonian becomes

$$
\begin{aligned}
H_{\mathrm{int}}= & g\left(2 \sum_{n=1}^{\infty} L_{-n} L_{n}+L_{0}^{2}-\frac{c+2}{12} L_{0}+\frac{c(22+5 c)}{2880}\right) \\
& +\bar{g}\left(2 \sum_{n=1}^{\infty} \bar{L}_{-n} \bar{L}_{n}+\bar{L}_{0}^{2}-\frac{c+2}{12} \bar{L}_{0}+\frac{c(22+5 c)}{2880}\right) .
\end{aligned}
$$

Note that (3.4) is a combination of higher integrals of motion $I_{3}$ and $\bar{I}_{3}$ [18-20]

$$
H_{\text {int }}=g I_{3}+\bar{g} \bar{I}_{3}
$$

Remined also that the unperturbed Hamiltonian coincides with

$$
H_{0}=L_{0}+\bar{L}_{0}-\frac{c}{12} \equiv I_{1}+\bar{I}_{1}
$$

Since the commutator $\left[I_{1}, I_{3}\right]=0$, the perturbation theory appears to be remarkably simple. In fact, the calculations presented in the remaining part of the paper, strongly suggest that eigenstates of the transfer-matrix (2.4) simultaneously diagonalize all operators $I_{1}$, $I_{3}, \bar{I}_{1}$ and $\bar{I}_{3}$. It is expected that this feature is generic, and higher order calculations would involve further integrals of motion $I_{5}, I_{7}, \ldots$ as well, thus making contact between integrable structures of the lattice model and CFT (see [21] for discussion of the dimer model from this perspective).

Representation of the interaction Hamiltonian in form (3.4) is very convenient for calculation of various matrix elements. In particular it is straightforward (for arbitrary $c$ ) to get:

$$
\frac{\left\langle\Delta ; \bar{\Delta}\left|L_{1}^{p} \bar{L}_{1}^{\tilde{p}} H_{\text {int }} L_{-1}^{p} \bar{L}_{-1}^{\tilde{p}}\right| \Delta ; \bar{\Delta}\right\rangle}{\left\langle\Delta ; \bar{\Delta}\left|L_{1}^{p} \bar{L}_{-1}^{\tilde{p}} L_{-1}^{p} \bar{L}_{-1}^{\tilde{p}}\right| \Delta ; \bar{\Delta}\right\rangle}=g M(\Delta, p)+\bar{g} M(\bar{\Delta}, \tilde{p})
$$

where

$$
M(\Delta, p)=\left(\frac{c}{24}\right)^{2}+\frac{11 c}{1440}+(\Delta+p)\left(-\frac{c}{12}+\Delta+\frac{p(2 \Delta+p)(5 \Delta+1)}{(\Delta+1)(2 \Delta+1)}-\frac{1}{6}\right)
$$

This result was found by a different technique long ago in [22]. Another set of matrix elements for Ising model will be $[23,24]$ (now the central charge is specified to $c=1 / 2$ )

$$
\frac{\left\langle\frac{1}{16} ; \frac{1}{16}\left|\psi_{p} H_{\text {int }} \psi_{-p}\right| \frac{1}{16} ; \frac{1}{16}\right\rangle}{\left\langle\frac{1}{16} ; \frac{1}{16}\left|\psi_{p} \psi_{-p}\right| \frac{1}{16} ; \frac{1}{16}\right\rangle}=g M(p)+\bar{g} M(0)
$$


where

$$
M(p)=\frac{7 p^{3}}{6}-\frac{7}{1440}
$$

\subsection{Identification of coupling constants}

Comparing the leading term of (2.13) with (2.10) and taking into account that for vacuum state $\Delta=\bar{\Delta}=0$, we easily verify that the values of parameters $q, \bar{q}$ are given by eq. (2.11) and that central charge $c=\frac{1}{2}$. It is convenient to represent (2.13) (included the second, next to leading term) as (c.c. stands for complex conjugate)

$$
\begin{aligned}
& \frac{\pi \sin (4 u)}{12 N}+\frac{7 \pi^{3}\left(2 \sin (4 u)-4 \sin ^{3}(4 u)\right)}{1440 N^{3}} \\
& =\left(-\frac{1}{48}+\frac{7 \pi^{2}}{5760 N^{2}}\left(1-e^{-8 i u}\right)\right) \frac{\log q}{M}+\text { c.c. }
\end{aligned}
$$

The term

$$
\delta E_{0}=\frac{7 \pi^{2}}{5760 N^{2}}\left(1-e^{-8 i u}\right)
$$

can be interpreted as a shift of (holomorphic part of) the vacuum state energy due to perturbation by $H_{\mathrm{int}}$. This leads to the relation

$$
g M(0,0)=\frac{7 \pi^{2}}{5760 N^{2}}\left(1-e^{-8 i u}\right)
$$

Since, due to (3.6)

$$
M(0,0)=\frac{c(5 c+22)}{2880}=\frac{49}{11520}
$$

for the coupling constant we get

$$
g=\frac{2 \pi^{2}}{7 N^{2}}\left(1-e^{-8 i u}\right)
$$

Analogously, the conjugate coupling

$$
\bar{g}=\frac{2 \pi^{2}}{7 N^{2}}\left(1-e^{8 i u}\right) .
$$

Now let us consider in some details the isotropic case $u=\frac{\pi}{8}$. In this case we have

$$
g=\bar{g}=\frac{4 \pi^{2}}{7 N^{2}}
$$

It is subtle but possible to compare this result with the coupling constant

$$
g_{l}=-\frac{1}{28 \pi},
$$

obtained in [25] for the (not rotated) square lattice critical Ising model. To make a correct comparison one should take into account that in current paper we have chosen a natural from CFT point of view energy normalization such that the level spacing in leading order 
is equal to 1 , while in [25] it is equal to $\frac{\tilde{N}}{2 \pi}$ (to avoid confusion we denote the parameters $N, M$, of [25] by $\tilde{N}, \tilde{M})$. Besides, $\tilde{N}$ should be rescaled by the geometric factor $\sqrt{2}$ (since the number of sites $\frac{N M}{2}$ should be identified with $\left.\tilde{N} \tilde{M}\right)$. Finally a factor -1 arises due to rotation of lattice by $\theta=\frac{\pi}{4}$. Indeed, the spin of the perturbing field is $s=4$, hence we get the phase factor

$$
e^{i \theta s}=-1 .
$$

Carefully picking up all the factors arising from above considerations one arrives at the consistency condition

$$
g=-\frac{(2 \pi)^{3}}{\tilde{N}^{2}} g_{l}
$$

which, obviously is satisfied.

In what follows, besides the vacuum state, we'll examine infinitely many other states and will demonstrate how the values (3.12), (3.13) of the coupling constants reproduces correct energy shifts.

\subsection{Energy corrections for the states $L_{-1}^{p-1} \bar{L}_{-1}^{\tilde{p}-1}|1 / 2 ; 1 / 2\rangle$}

It is straightforward to deduce from (2.16), (2.13) that the large $N$ expansion of the transfermatrix eigenvalues in $r=1$ sector with two sign flips $\mu_{p}=-1$ and $\bar{\mu}_{\tilde{p}}=-1$ can be represented as

$$
\begin{aligned}
M \log \Lambda_{p, \tilde{p}}^{(+)} & +\frac{N M}{2} f_{\mathrm{bulk}} \\
= & \left(-\frac{1}{48}+\frac{1}{2}+p-1+\frac{\pi^{2}\left(1-e^{-8 i u}\right)}{5760 N^{2}}\left(7+1920(p-1 / 2)^{3}\right)\right) \log q \\
& +\left(-\frac{1}{48}+\frac{1}{2}+\tilde{p}-1+\frac{\pi^{2}\left(1-e^{8 i u}\right)}{5760 N^{2}}\left(7+1920(\tilde{p}-1 / 2)^{3}\right)\right) \log \bar{q} \\
& +O\left(1 / N^{5}\right)
\end{aligned}
$$

The leading terms in above expressions are displayed in such specific way to emphasize the structure

$$
-\frac{c}{24}+\Delta+l
$$

where $\Delta$ is the dimension of respective primary field and $l$ is the excitation level. According to eq. (2.23), the states under consideration should be identified with $\psi_{-p+\frac{1}{2}} \bar{\psi}_{-\tilde{p}+\frac{1}{2}}|0 ; 0\rangle$. Using commutation relation

$$
\left[L_{n}, \psi_{\nu}\right]=-\left(\frac{n}{2}+\nu\right) \psi_{n+\nu}
$$

it is easy to show that

$$
L_{-1}^{p} \bar{L}_{-1}^{\tilde{p}}|1 / 2 ; 1 / 2\rangle \sim \psi_{-p+\frac{1}{2}} \bar{\psi}_{-\tilde{p}+\frac{1}{2}}|0 ; 0\rangle
$$

Comparing (3.5) with subleading terms of (3.14) we see that consistency with perturbation theory requires the equality

$$
g M(1 / 2, p)=\frac{\pi^{2}\left(1-e^{-8 i u}\right)}{5760 N^{2}}\left(7+1920(p-1 / 2)^{3}\right)
$$

which is easily checked to be satisfied identically due to (3.12) and (3.6). 


\subsection{Energy corrections for the states $\psi_{-p} \bar{\psi}_{-\tilde{p}}|1 / 16 ; 1 / 16\rangle$}

In this section we will examine the analogues infinite series of states in $r=-1$ sector. Again we will assume that $\mu_{p}=-1, \bar{\mu}_{\tilde{p}}=-1$ and all other $\mu, \bar{\mu}=1$. In this case we get the large $N$ expansion

$$
\begin{aligned}
M \log \Lambda_{p, \tilde{p}}^{(-)} & +\frac{N M}{2} f_{\text {bulk }}= \\
= & \left(-\frac{1}{48}+\frac{1}{16}+p+\frac{\pi^{2}\left(1-e^{-8 i u}\right)}{720 N^{2}}\left(-1+240 p^{3}\right)\right) \log q \\
& +\left(-\frac{1}{48}+\frac{1}{16}+\tilde{p}+\frac{\pi^{2}\left(1-e^{8 i u}\right)}{720 N^{2}}\left(-1+240 \tilde{p}^{3}\right)\right) \log \bar{q} \\
& +O\left(1 / N^{5}\right)
\end{aligned}
$$

In this case the perturbation theory requires (see (3.7) and 2.17):

$$
g M(p)=\frac{\pi^{2}\left(1-e^{-8 i u}\right)}{720 N^{2}}\left(-1+240 p^{3}\right)
$$

which again is satisfied identically due to (3.12) and (3.8).

\subsection{An example of doubly degenerate states}

Now let us consider a case, when double degeneracy takes place. Namely we will consider two forth level states

$$
|1\rangle \equiv \psi_{-7 / 2} \psi_{-1 / 2}|0 ; 0\rangle ; \quad|2\rangle \equiv \psi_{-5 / 2} \psi_{-3 / 2}|0 ; 0\rangle .
$$

Though not obvious, a direct calculation, sketched below, shows that the matrix element of $H_{\text {int }}$ between these states is zero, and that the energy shifts for either of these states indeed agree with lattice prediction.

To make actual calculations, let us remind that

$$
T(z)=-\frac{1}{2}: \psi(z) \partial \psi(z):
$$

where :: stands for normal ordering. In terms of modes this is equivalent to

$$
L_{k}=\frac{1}{4} \sum_{\nu+\mu=k}(\mu-\nu): \psi_{\nu} \psi_{\mu}:
$$

As an example let us calculate the action of $L_{-2}^{2}$ on vacuum state:

$$
\begin{aligned}
L_{-2}^{2}|0 ; 0\rangle & =\left(\cdots \psi_{-\frac{7}{2}} \psi_{\frac{3}{2}}+\psi_{-\frac{5}{2}} \psi_{\frac{1}{2}}+\psi_{-\frac{3}{2}} \psi_{-\frac{1}{2}}\right)\left(\cdots+\psi_{-\frac{5}{2}} \psi_{\frac{1}{2}}+\psi_{-\frac{3}{2}} \psi_{-\frac{1}{2}}\right)|0 ; 0\rangle \\
& =\frac{5}{4} \psi_{-\frac{7}{2}} \psi_{-\frac{1}{2}}|0 ; 0\rangle-\frac{3}{4} \psi_{-\frac{5}{2}} \psi_{-\frac{3}{2}}|0 ; 0\rangle \equiv \frac{5}{4}|1\rangle-\frac{3}{4}|2\rangle
\end{aligned}
$$

Similarly we get

$$
L_{-1}^{2} L_{-2}|0 ; 0\rangle=3|1\rangle+|2\rangle \text {. }
$$


Inverting the relations (3.23) and (3.24) we obtain

$$
\begin{aligned}
& |1\rangle=\frac{2}{7} L_{-2}^{2}|0 ; 0\rangle+\frac{3}{14} L_{-1}^{2} L_{-2}|0 ; 0\rangle, \\
& |2\rangle=-\frac{6}{7} L_{-2}^{2}|0 ; 0\rangle+\frac{5}{14} L_{-1}^{2} L_{-2}|0 ; 0\rangle .
\end{aligned}
$$

Thus calculation of matrix elements of the interaction Hamiltonian (3.4) boils down to simple Virasoro algebra manipulations. Here are the results of calculations:

$$
\begin{aligned}
& \left\langle 1\left|H_{\text {int }}\right| 1\right\rangle=\frac{577969}{11520} g+\frac{49}{11520} \bar{g} \\
& \left\langle 2\left|H_{\text {int }}\right| 2\right\rangle=\frac{255409}{11520} g+\frac{49}{11520} \bar{g} \\
& \left\langle 1\left|H_{\text {int }}\right| 2\right\rangle=\left\langle 2\left|H_{\text {int }}\right| 1\right\rangle=0
\end{aligned}
$$

According to the rules established in subsection 2.4 the state $|1\rangle$ corresponds to the transfermatrix eigenvalue with sign flips $\mu_{1}=-1, \mu_{4}=-1$ and $|2\rangle$ corresponds to $\mu_{2}=-1$, $\mu_{3}=-1$ (both states belong to the sector $r=1$ ). From (2.16), (2.13) we get expansions

$$
\begin{aligned}
M \log \Lambda_{1,4}^{(+)} & +\frac{N M}{2} f_{\text {bulk }} \\
= & \left(-\frac{1}{48}+4+\frac{\pi^{2}\left(1-e^{-8 i u}\right)}{N^{2}}\left(\frac{7}{5760}+\frac{1}{3}\left((1-1 / 2)^{3}+(4-1 / 2)^{3}\right)\right)\right) \log q \\
& +\left(-\frac{1}{48}+\frac{7 \pi^{2}\left(1-e^{8 i u}\right)}{5760 N^{2}}\right) \log \bar{q}+O\left(1 / N^{5}\right) \\
M \log \Lambda_{2,3}^{(+)} & +\frac{N M}{2} f_{\text {bulk }}= \\
= & \left(-\frac{1}{48}+4+\frac{\pi^{2}\left(1-e^{-8 i u}\right)}{N^{2}}\left(\frac{7}{5760}+\frac{1}{3}\left((2-1 / 2)^{3}+(3-1 / 2)^{3}\right)\right)\right) \log q \\
& +\left(-\frac{1}{48}+\frac{7 \pi^{2}\left(1-e^{8 i u}\right)}{5760 N^{2}}\right) \log \bar{q}+O\left(1 / N^{5}\right)
\end{aligned}
$$

The expressions (3.27), (3.28) are in perfect agreement with (3.26), since the relations

$$
\begin{aligned}
\frac{577969}{11520} g & =\frac{\pi^{2}\left(1-e^{-8 i u}\right)}{N^{2}}\left(\frac{7}{5760}+\frac{1}{3}\left((1-1 / 2)^{3}+(4-1 / 2)^{3}\right)\right) \\
& =\frac{82567}{5760} \frac{\pi^{2}\left(1-e^{-8 i u}\right)}{N^{2}}, \\
\frac{255409}{11520} g & =\frac{\pi^{2}\left(1-e^{-8 i u}\right)}{N^{2}}\left(\frac{7}{5760}+\frac{1}{3}\left((2-1 / 2)^{3}+(3-1 / 2)^{3}\right)\right) \\
& =\frac{36487 \pi^{2}\left(1-e^{-8 i u}\right)}{5760} \frac{N^{2}}{49} \bar{g} \\
\overline{11520} & =\frac{7 \pi^{2}\left(1-e^{8 i u}\right)}{5760 N^{2}}
\end{aligned}
$$

in view of (3.12), are satisfied identically. 


\section{Summary and discussion}

To summarize let us quote the main results of this paper:

- The leading irrelevant perturbation (3.4), which controls the deviation of critical lattice Ising model with periodic boundary conditions from its continuous CFT analog is identified. The relation (3.12) between anisotropy parameter and the coupling constant is established

- Next to leading $\sim 1 / N^{2}$ corrections to the spectrum are calculated independently from lattice theory and from the perturbed CFT for several classes of states always finding exact agreement (subsections 3.2-3.4).

- It is expected that to mimic higher order corrections, one should add to unperturbed Hamiltonian terms, proportional to the higher integrals of motion in Ising CFT. In this way the integrable structure of the lattice theory gets related to the integrable structure of CFT.

Besides square lattice, 2d Ising model is exactly solvable also on a number of other lattices with different symmetries. It would be interesting to identify the corresponding perturbing fields also in these cases. Clearly, it is expected that the spin of a perturbing field should be consistent with the order of discrete rotations allowed by respective lattice symmetry.

It must be also possible to extend our analysis to the entire spectrum and higher orders in $1 / N$ expansion, but this is left for future work.

\section{Acknowledgments}

I am grateful to my supervisor Prof. Nikolay Izmailyan and Prof. Rubik Poghossian for introducing me into this field and for stimulating discussions.

This work was partially supported by the Armenian State Committee of Science in the framework of the research project 18T-1C113.

Open Access. This article is distributed under the terms of the Creative Commons Attribution License (CC-BY 4.0), which permits any use, distribution and reproduction in any medium, provided the original author(s) and source are credited.

\section{References}

[1] J.G.E. Brezin and J.Zinn-Justin, Field theoretical approach to critical phenomena, in Phase transitions and critical phenomena. Volume 6, C. Domb and J.L. Lebowitz eds., Academic Press, New York U.S.A. (1972).

[2] J. Cardy, Conformal invariance, in Phase transitions and critical phenomena. Volume 11, C. Domb and J. L. Lebowitz eds., Academic Press, New York U.S.A. (1987).

[3] J.L. Cardy, Effect of boundary conditions on the operator content of two-dimensional conformally invariant theories, Nucl. Phys. B 275 (1986) 200 [INSPIRE].

[4] E. Ising, Contribution to the theory of ferromagnetism, Z. Phys. 31 (1925) 253 [INSPIRE]. 
[5] L. Onsager, Crystal statistics. 1. A Two-dimensional model with an order disorder transition, Phys. Rev. 65 (1944) 117 [INSPIRE].

[6] R. Baxter, Exactly solved models in statistical mechanics, Dover books on physics, Dover Publications, U.S.A. (2007).

[7] D.L. O'Brien, P.A. Pearce and S.O. Warnaar, Finitized conformal spectrum of the Ising model on the cylinder and torus, Physica A 228 (1996) 63.

[8] R.E. Behrend, P.A. Pearce and D.L. O'Brien, Interaction-round-a-face models with fixed boundary conditions: The ABF fusion hierarchy, J. Statist. Phys. 84 (1996) 1 [hep-th/9507118] [INSPIRE].

[9] R.E. Behrend, P.A. Pearce, V.B. Petkova and J.-B. Zuber, Boundary conditions in rational conformal field theories, Nucl. Phys. B 570 (2000) 525 [hep-th/9908036] [INSPIRE].

[10] C. Chui, C. Mercat, W. P. Orrick and P.A. Pearce, Integrable lattice realizations of conformal twisted boundary conditions, Phys. Lett. B 517 (2001) 429.

[11] C.H.O. Chui, C. Mercat and P.A. Pearce, Integrable and conformal twisted boundary conditions for $\mathrm{sl}(2)$ A-D-E lattice models, J. Phys. A 36 (2003) 2623 [hep-th/0210301] [INSPIRE].

[12] H.W.J. Bloete, J.L. Cardy and M.P. Nightingale, Conformal invariance, the central charge and universal finite size amplitudes at criticality, Phys. Rev. Lett. 56 (1986) 742 [InSPIRE].

[13] I. Affleck, Universal term in the free energy at a critical point and the conformal anomaly, Phys. Rev. Lett. 56 (1986) 746 [INSPIRE].

[14] J. Salas and A.D. Sokal, Universal amplitude ratios in the critical two-dimensional Ising model on a torus, J. Statist. Phys. 98 (2000) 551 [cond-mat/9904038] [INSPIRE].

[15] P. Di Francesco, P. Mathieu and D. Sénéchal, Conformal field theory, Graduate Texts in Contemporary Physics, Springer, Germany (1997).

[16] J.L. Cardy, Operator content of two-dimensional conformally invariant theories, Nucl. Phys. B 270 (1986) 186 [INSPIRE].

[17] A.B. Zamolodchikov, Renormalization group and perturbation theory near fixed points in two-dimensional field theory, Sov. J. Nucl. Phys. 46 (1987) 1090 [InSPIRE].

[18] R. Sasaki and I. Yamanaka, Virasoro algebra, vertex operators, quantum sine-Gordon and solvable quantum field theories, Adv. Stud. Pure Math. 16 (1988) 271.

[19] T. Eguchi and S.-K. Yang, Deformations of conformal field theories and soliton equations, Phys. Lett. B 224 (1989) 373 [INSPIRE].

[20] V.V. Bazhanov, S.L. Lukyanov and A.B. Zamolodchikov, Integrable structure of conformal field theory, quantum KdV theory and thermodynamic Bethe ansatz, Commun. Math. Phys. 177 (1996) 381 [hep-th/9412229] [INSPIRE].

[21] A. Morin-Duchesne, J. Rasmussen and P. Ruelle, Integrability and conformal data of the dimer model, J. Phys. A 49 (2016) 174002 [arXiv:1507.04193] [INSPIRE].

[22] P. Reinicke, Analytical and nonanalytical corrections to finite size scaling, J. Phys. A 20 (1987) 5325 [INSPIRE].

[23] A. Poghosyan, R. Kenna and N. Izmailian, The critical ising model on a torus with a defect line, Europhys. Lett. 111 (2015) 60010. 
[24] A. Poghosyan, N. Izmailian and R. Kenna, Exact solution of the critical Ising model with special toroidal boundary conditions, Phys. Rev. E 96 (2017) 062127 [arXiv:1610.07855] [INSPIRE].

[25] N.S. Izmailian and C.-K. Hu, Exact universal amplitude ratios for two-dimensional ising models and a quantum spin chain, Phys. Rev. Lett. 86 (2001) 5160. 